Department of Infectious and Tropical Diseases, London School of Hygiene and Tropical Medicine, London

WC1E 7HT

Adriana Tami clinical lecturer

University of Nottingham and

Nottingham Health Authority

Richard Slack consultant for diseases control

Correspondence to: H Grundmann hajo.grundmann@ nottingham.ac.uk and the likelihood of finding healthy elderly people colonised with MRSA is relatively small. Carriage of MRSA can, however, be expected in patients who have chronic conditions or who have had recent admissions to hospital. MRSA in the community seems to be a consequence of a "spill over" of an uncontrolled hospital epidemic, and the few isolates found in the community are classic hospital strains. It continues to be safe to treat community acquired $S$ aureus infections in England with conventional antistaphylococcal agents effective against methicillin sensitive strains, and third line antibiotics should be considered only when typical risk factors can be ascertained.

Contributors: HG designed and coordinated the study. AT took part in every aspect of the study and carried out the statistical analysis. SH and MH carried out the bacteriological investigations and the genetic typing. All authors were involved in collecting samples and recording data. RS coordinated the general practitioner and community participation. HG wrote the manuscript and was supported by AT and RS in the final draft. $\mathrm{HG}$ and $\mathrm{RS}$ are the guarantors.

Funding: The study was supported by a grant from Nottingham Health Authority.

Competing interests: None declared.

1 Public Health Laboratory Service. Epidemic methicillin-resistant Staphy lococcus aureus. Commun Dis Rep CDR Wkly 1997;7:191.

2 Chambers HF. The changing epidemiology of Staphylococcus aureus? Emerg Infect Dis 2001;7:178-82.

3 Hori S, Sunley R, Tami A, Grundmann H. The Nottingham Staphylococcus aureus population study: prevalence of MRSA among the elderly in a university hospital. J Hosp Infect 2002;50:25-9.

4 Nottingham Health Authority. Aiming for health in the year 2000 Annual report of the Director of Public Health. Nottingham: Nottingham Health Authority, 1995:3-11

5 Jarman B. Identification of underprivileged areas. BMJ 1983;286:1705-9. (Accepted 3 December 2001)

\title{
Longitudinal study of genital infection by herpes simplex virus type 1 in western Scotland over 15 years
}

\author{
Anne Scoular, John Norrie, Graeme Gillespie, Noreen Mir, W F Carman
}

Department of Genitourinary

Medicine,

Sandyford Initiative,

Glasgow G3 7NB

Anne Scoular

consultant physician

Robertson Centre

for Biostatistics,

University of

Glasgow, Glasgow

G12 8QQ

John Norrie

assistant director

West of Scotland

Specialist Virology

Centre, Gartnavel

General Hospital,

Glasgow G12 0XN

W F Carman

director

Graeme Gillespie

biomedical scientist

Department of

Genitourinary

Medicine, Russell

Institute, Paisley

PA1 1UR

Noreen Mir

consultant physician

Correspondence to:

A Scoular

anne@scoular.

demon.co.uk

BMJ 2002;324:1366-7
Although herpes simplex virus type 2 (HSV-2) is regarded as causing most cases of genital herpes, preliminary reports suggest that the type 1 virus (HSV-1) is increasingly the cause of infection. ${ }^{1}$ Recurrence rates, viral shedding, and the mode of acquiring HSV-1 infection are different from those for HSV-2, so counselling and clinical management strategies may need to be revised. We studied longitudinal trends in laboratory reports of genital HSV-1 infection.

\section{Methods and results}

The West of Scotland Specialist Virology Centre processes $99 \%$ of all herpes simplex virus culture samples in the region. All genital samples of herpes simplex processed between 1 January 1986 and 31 December 2000 were reviewed for source of referral, patient's sex and age (stratified into seven bands: $\leqslant 20,21-25,26-30$, $31-35,36-40,41-45$, and $>45$ years), and the type of virus isolated.

Samples were cultured and then typed using fluorescein labelled monoclonal antibodies to HSV-1 and HSV-2 (Syva Microtrak). From January 1999, the virus was detected and typed using a polymerase chain reaction method and restriction fragment length polymorphism. ${ }^{2}$ The referral patterns and age and sex profiles of patients did not change during the period of analysis.

We compared the proportion of HSV-1 in all positive swabs between sexes and ages using $\chi^{2}$ tests, and over the three year time bands by the Cochran Armitage trend test (both overall and within four subgroups with age categorised as $\leqslant 25$ years or $>25$ years for each sex) using SAS 8.2.

Of 10547 swabs, the virus was identified in 3181 (30\%); 3126 were typed, $1530(49 \%)$ as HSV-1 and
$1596(51 \%)$ as HSV-2. Of the swabs testing positive for HSV, 2004 (63\%) were from women and 1177 (37\%) were from men. Age was recorded for 3099 (97.4\%) patients, with $555(18 \%)$ aged $\leqslant 20,885(29 \%)$ aged 21-25, $686(22 \%)$ aged 26-30, $413(13 \%)$ aged 31-35, $239(8 \%)$ aged 36-40, $159(5 \%)$ aged 41-45, and 162 $(5 \%)$ aged $>45$ years. The origin of the request to detect the virus was recorded for 10476 (99\%) samples: $7579(72 \%)$ were from genitourinary medicine clinics, $678(6 \%)$ from general practice, $223(2 \%)$ from family planning clinics, and 1996 (19\%) from other sources.

HSV-1 was strongly associated with female sex and younger age $(\mathrm{P}<0.0001)$. Over the entire study period,

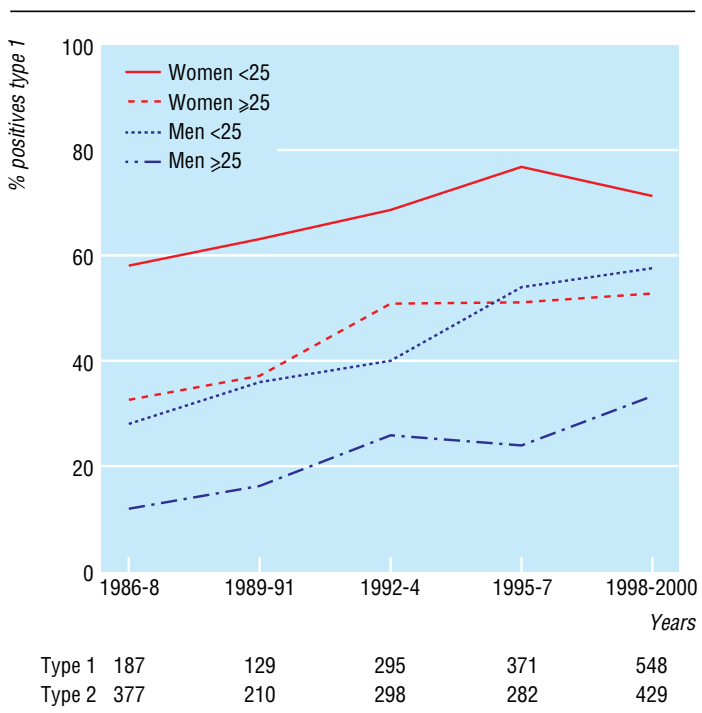

Proportion (\%) of herpes simplex virus test that were type 1 
HSV-1 was found in $751(70 \%)$ of all positive swabs in women $<25$ years, $141(41 \%)$ in men $<25$ years, 413 $(49 \%)$ in women $\geqslant 25$ years, and $182(23 \%)$ in men $\geqslant 25$ years.

In 1986-8, 33\% (187) of all positive swabs were due to $\mathrm{HSV}-1$, rising progressively to $56 \%$ (548) in $1998-2000(\mathrm{P}<0.0001)$. A significant rise $(\mathrm{P}<0.0001$, $1986 v 2000)$ in the proportion of isolates attributable to HSV-1 occurred in each of the four age and sex subgroups $(\mathrm{P}<0.0001)$ (figure).

\section{Comment}

Both the number and percentage of genital HSV-1 infections have risen. Genital infection with HSV-1 is strongly associated with being young (aged $<25$ years) and being female.

Explanations include changing host susceptibility and changing sexual behaviour of the population. The population seroprevalence of $\mathrm{HSV}-1$ is falling: increasing numbers of young adults are susceptible to HSV-1 infection. ${ }^{3}$ As genital tract reactivation of latent HSV-1 infection is infrequent, most new cases of genital HSV-1 infection are likely to be due to orogenital transmission, but there is no evidence suggesting that oral sex practices have changed substantially. ${ }^{4}$ The occurrence of HSV-1 infection in women, seen consistently in other studies, ${ }^{1}$ is unexplained.

These results have three important implications for management. Firstly, patients should be counselled about the more favourable clinical course of genital HSV-1 than of HSV-2 infection; recurrences are generally milder and infrequent. Secondly, subclinical shedding of HSV-1 is less common; this has a direct bearing on the likelihood of transmission. ${ }^{5}$ Thirdly, preventive strategies for genital herpes should focus on the risk of unprotected orogenital intercourse, which is frequently perceived as "safe" in the context of sexually transmitted infections.

We thank Geoffrey Clements, previously director of the West of Scotland Specialist Virology Centre.

Contributors: AS initiated and designed the study, analysed and interpreted the results, and wrote the paper. JN and NM analysed and interpreted the results and wrote the paper. GG contributed to the study design and analysis of results. WC contributed to the study design, analysis, and interpretation of the results. AS is guarantor.

Funding: No additional funding.

Competing interests: None declared.

1 Lamey P-J, Hyland PL. Changing epidemiology of herpes simplex virus type 1 infections. Herpes 1999;6:20-4.

2 Scoular A, Gillespie G, Carman WF. Polymerase chain reaction for diagnosis of genital herpes in a genitourinary medicine clinic. Sex Transm Infect 2002;78:21-5.

3 Vyse AJ, Gay NJ, Slomka M, Gopal R, Gibbs T, Morgan-Capner P, et al. The burden of infection with HSV-1 and HSV-2 in England and Wales: implications for the changing epidemiology of genital herpes. Sex Transm Infect 2000;76:183-7.

4 Johnson AM, Wadsworth J, Wellings K, Field J. Sexual attitudes and lifestyles. Oxford: Blackwell Scientific, 1994

5 Lafferty WE, Coombs RW, Benedetti J, Critchlow C, Corey L. Recurrences after oral and genital herpes simplex virus infection: influence of site of infection and viral type. N Engl J Med 1987;316:1444-9.

(Accepted 3 December 2001)

\title{
Follow up after a family based genetic screening programme for familial hypercholesterolaemia: is screening alone enough?
}

\author{
Merel C van Maarle, Marlies E A Stouthard, Perla J Marang-van de Mheen, Niek S Klazinga, \\ Gouke J Bonsel
}

Familial hypercholesterolaemia is an autosomal dominant disorder of lipoprotein metabolism, with an estimated frequency of 1 in 500 in Western countries; it results in excess mortality from coronary artery disease. ${ }^{1}$ Now that the genetic defects can be detected and statins are available to lower lipids effectively, genetic screening has been considered. ${ }^{2-3}$ In 1994 a family based genetic screening programme for familial hypercholesterolaemia started in the Netherlands. The programme's effectiveness rests on the evidence based treatment of newly identified patients. We therefore assessed the subsequent preventive care and short term clinical outcome in people testing positive for familial hypercholesterolaemia as a proxy for the expected long term level of coronary artery disease. ${ }^{4}$

\section{Participants, methods, and results}

The foundation for tracing hereditary hypercholesterolaemia performs cascade screening in families of patients with clinically diagnosed familial hypercholes- terolaemia with a known mutation, actively approaching first degree and second degree relatives. ${ }^{5}$ Family members are tested for the known mutation; their cholesterol level is not measured. The test result is communicated only to the person screened (by mail). The foundation is not involved in subsequent treatment or in monitoring follow up.

We conducted the evaluation study from March to September 1998 in all 215 people who tested positive from a consecutive cohort of 677 people screened as part of the programme. The inclusion criteria were consent to genetic testing and the current study, a positive test result, and age 18 or over.

We collected data with three self administered questionnaires-at screening and at 7 months and 18 months after communication of the test result. The main outcome measures were quality of treatment according to the key recommendations of the Dutch guidelines on hypercholesterolaemia and quality of clinical outcome by achieved cholesterol level, body mass index, and smoking status (table). ${ }^{4}$
Department of Social Medicine, Academic Medical Centre, University of Amsterdam, PO Box 22700, 1100 DE Amsterdam, Netherlands

Merel C van Maarle physician

Marlies E A Stouthard psychologist Niek S Klazinga professor in social medicine Gouke J Bonsel professor in public health methods

continued over

BMJ 2002;324:1367-8 